\title{
Micro-mechanical Pendulum and Application Technology in The Attitude Control System of The Rotating Body
}

Fuxue Zhang ${ }^{1, \mathrm{a}}$,Zengping Zhang ${ }^{2, \mathrm{~b}}$, Qingwen Yan ${ }^{2, \mathrm{c}}$, Hui Zhao ${ }^{2, \mathrm{~d}}$, Wei Zhang $^{1, e}$

${ }^{1}$ Sensing Technique Research Center, Beijing Information Science and Technology University, Beijing 100101, China

${ }^{2}$ School of Electronic Engineering, Beijing University of Posts and Telecommunications, Beijing 100876, China

azhangfuxue@263.net, 'bhangzp2007@hotmail.com, '16049403@qq.com, dasdfzh@163.com, eway_zh@163.com

Keywords: micromechanical pendulum; angular velocity; rotating bomb; gyro.

\begin{abstract}
Author found that the micro-mechanical pendulum mounted on a rotating body has gyroscopic effect, which can be sensitive to yaw, pitch and spin angular velocity of the rotating body at same time. It has the function of the three traditional gyro. This paper reported the principle of micro-mechanical pendulum structure, the gyroscopic effect in the rotating body, and sensitive to the rotating body attitude mechanism, as well as application in the attitude control system of the polar coordinate transformation. The tests showed that performance of the micro-mechanical pendulum for the rotating body attitude control system achieves the design requirements of attitude control.
\end{abstract}

\section{Introduction}

High-speed rotating body is widely used, but the bottleneck in the development is sensitive to rotating body's attitude sensor technology. This is the core technology involved in the practical use of the rotating body. However, it is not reported in the domestic and international so far.

The author found that the micro-mechanical pendulum mounted on the rotating body produces the gyroscopic effect due to the drive force generated by the spin of the rotating body. It can detect yaw, pitch and spin angular velocity of the rotating body, and it has the function of the three traditionalgyro ${ }^{[1,2]}$. Through application of this discovery the bottleneck is not only broken, but also the cost of using is greatly reduced ${ }^{[3 \sim 7]}$.

\section{Micro-mechanical pendulum}

Fig. 1(a) is a front view ${ }^{[4]}$ of the micro-mechanical pendulum. Fig. 1(b) is a signal acquisition circuit. Fig. 2 is a micro-mechanical pendulum chip's structural section.

Micro-mechanical pendulum was hanging in the box by the beam. Above and below the silicon pendulum chip, a pair of stopper pads is symmetrically set up. When the rotating body yaw or pitch, beam vibration put the pendulum piece along with vibration. Swing of the pendulum piece on intermediate layer leads to capacitance changes in the circuit. The signal outputs because of bridge out of balance. The detection signal can obtain the yaw, pitch and spin angular velocity. 

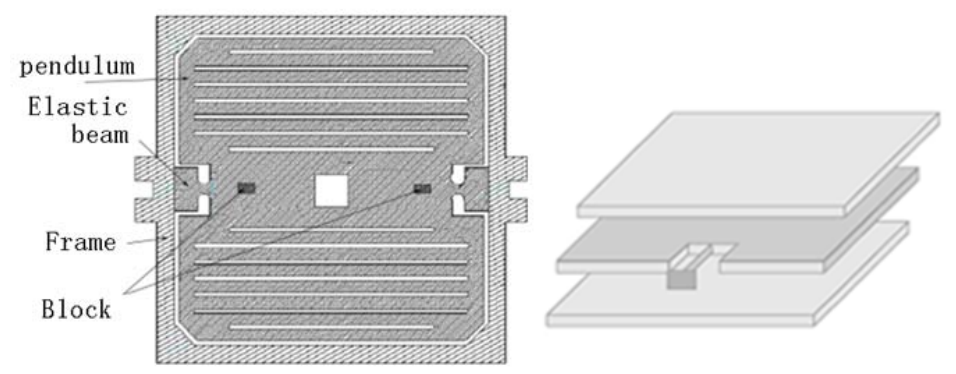

Fig.(a)Front view of the

micro-mechanical pendulum

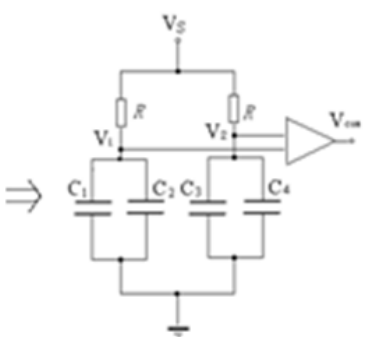

Fig. (b)Signal acquisition circuit

Fig.1 Front view of the micro-mechanical pendulum and signal acquisition circuit diagram

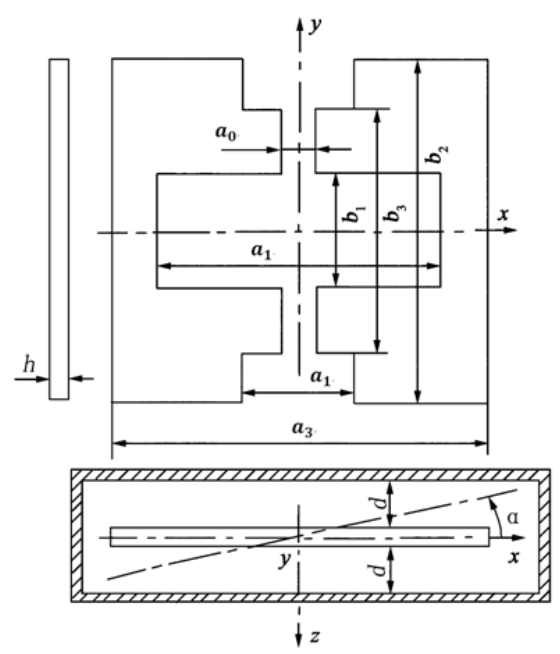

Fig.2 Micro-mechanical pendulum chip's structural section

\section{Sensitive to the rotating body's attitude mechanism}

Fig. 3 is a gyroscopic effect of the micro-mechanical pendulum mounted on the rotating body ${ }^{[3,5 \sim 7]}$. It is a sensitive schematic to rotating body yaw, pitch and spin angular velocity. The rotating body and micro-mechanical pendulum mounted on the rotating body constitute the closed-loop gyro together. The block diagram is shown in Fig. 4.

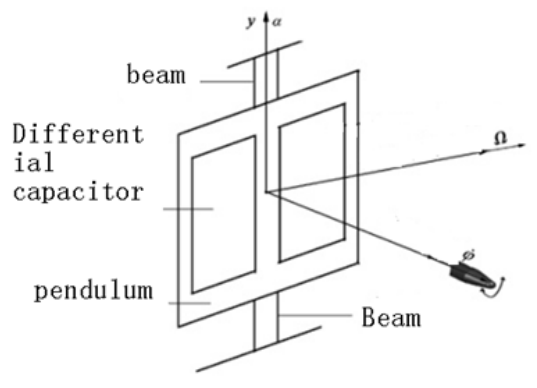

Fig.3 The shematic diagram of gyroscopic

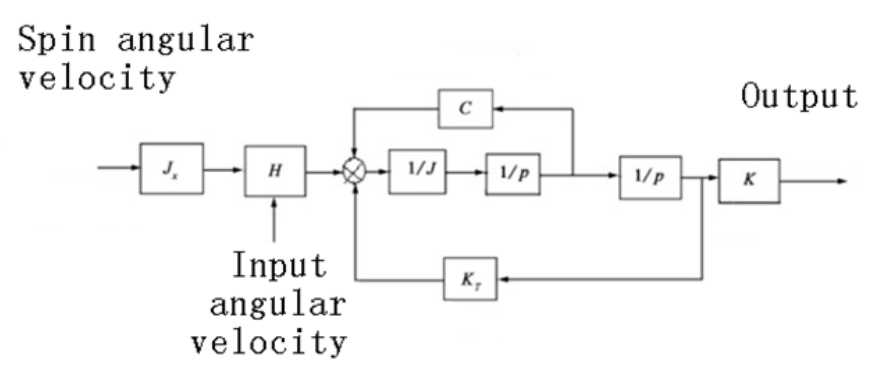

Fig.4 The shematic block diagram 
Fig. 4 shows a micro-mechanical pendulum can output the spin angular velocity and the transvers angular velocity (as polar). With geographic coordinate system, it output yaw, pitch and spin angular velocity.

Mounted on a rotating body, the micromechanical pendulum's kinematic equation is

$$
\begin{aligned}
& J_{y} \ddot{\alpha}+D \dot{\alpha}+\left[\left(J_{z}-J_{x}\right) \dot{\varphi}^{2}+K_{T}\right] \alpha \\
& =\left(J_{z}+J_{y}-J_{x}\right) \dot{\varphi} \Omega \sin \dot{\varphi} t
\end{aligned}
$$

Steady state solution:

$$
\alpha=\frac{\left(J_{z}+J_{y}-J_{x}\right) \dot{\varphi} \Omega}{\sqrt{\left[\left(J_{z}-J_{y}-J_{x}\right) \dot{\varphi}^{2}+K_{T}\right]^{2}+(D \dot{\varphi})^{2}}} \sin \dot{\varphi} t
$$

Eq. (2) shows that the output of micro-mechanical pendulum swing angle $\alpha$ is related to the spin angular velocity $\dot{\varphi}$ and the transvers angular velocity $\Omega$. The output waveforms are shown in Fig. 5 and Fig. 6.

The vertical axis represents the output voltage signal in Fig. 5, the horizontal axis represents time. The spin angular velocity is available from the carrier (Fig. 6 (a)), while the envelope is to enter the transverse angular velocity (Fig. 6 (b)). If the transverse angular velocity is constant, the envelope is a straight line (Fig. 6 (a)). With the geographical coordinate system, the output signal includes yaw, pitch and spin angular velocity.

The above characteristics show that micro-mechanical pendulum's structure is simple. It can also output the yaw, pitch, and the spin angular velocity, with three general gyro's function and the use of low cost.

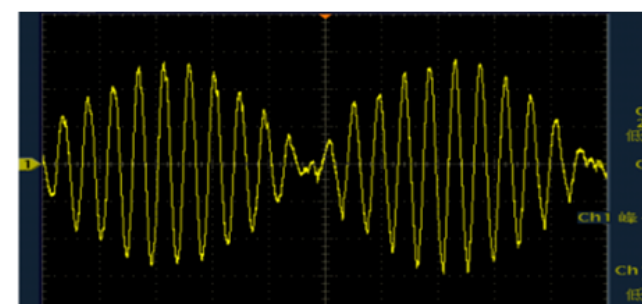

Fig.5 Micro-mechanical pendulum the output

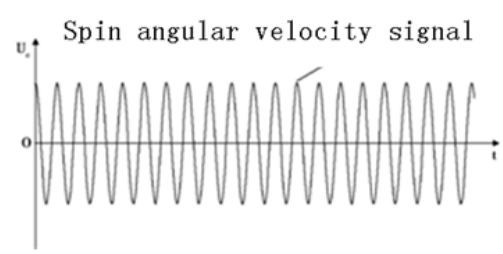

(a) Carrier signal

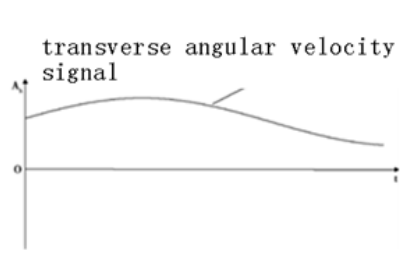

(b) The envelope
MEMS Output Signal

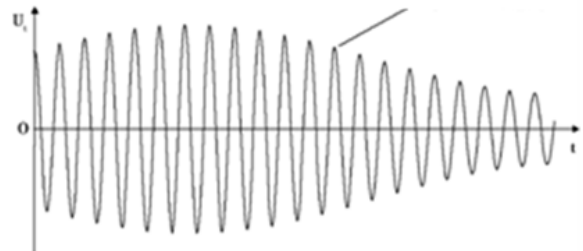

(c) AM signal

Fig.6 Carrier and envelope of output signal 
As shown in Fig. 7, $\mathrm{O}$ is a fixed point on the vertical axis of the rotating body and $\mathrm{OZ}$ fixes on the vertical axis of the rotating body. To take two points $M, N$ on the rotating body, make $O M \perp O N$ and surface $O M N \perp O Z$. The coordinate system $o x y z$ of the rotating body is established, of which the axis $x$ is same direction as $O M$, the axis $y$ is same direction as $O N$, and the axis $z$ is same direction as $O Z$.

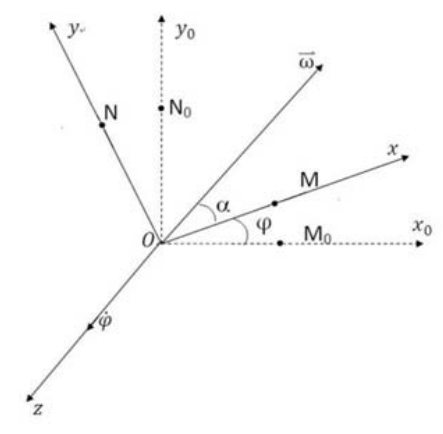

Fig.7 Rotating body coordinate system transformation

When the rotating body in Fig.7 rotates around the axis $\mathrm{z}$ with the spin angular velocity $\dot{\varphi}$, the coordinate plane will be the same around the axis $\mathrm{z}$ with angular velocity $\dot{\varphi}$. It is supposed that the points $M$ and Nare respectively $M_{0}$ of axis $O M_{0}$ and $N_{0}$ of axis $O N_{0}$ when rotating body began to turn. We establish coordinate system $\mathrm{ox}_{0} \mathrm{y}_{0} \mathrm{z}$ with $\mathrm{OM}_{0}, \mathrm{ON}_{0}, \mathrm{OZ}$, where $\mathrm{ox}_{0}$ and $\mathrm{OM}_{0}$ direction, oy and $\mathrm{ON}_{0}$ direction, and $\mathrm{OZ}$ and $\mathrm{OZ}$ direction are in the same direction.

We define the coordinate system $\mathrm{ox}_{0} \mathrm{y}_{0} \mathrm{z}$ subject to the rotating body coordinate system. Then oxyz and $0 x_{0} y_{0} z$ are the following relation.

$$
\left(\begin{array}{l}
x_{0} \\
y_{0} \\
z
\end{array}\right)=\left(\begin{array}{ccc}
\cos \varphi & -\sin \varphi & 0 \\
\sin \varphi & \cos \varphi & 0 \\
0 & 0 & 1
\end{array}\right)\left(\begin{array}{l}
x \\
y \\
z
\end{array}\right)
$$

In that coordinate system $\mathrm{ox}_{0} \mathrm{y}_{0} \mathrm{z}$ turn angle $\varphi(\mathrm{t})$ around the $\mathrm{z}$-axis with counterclockwise rotation, namely the rotating body coordinate system oxyz can be obtained.

Micro-mechanical pendulum A and B are installed as shown in Fig. (8). Their sensitive axes are perpendicular to each other. A sensitive axis is in the X-axis and B sensitive axis in the $y$-axis. Coordinate plane oxy of the coordinate system oxyz (see Fig.7) of the rotating body is fixed on micro-mechanical pendulum that is in the rotating body. The oxy rotates along with rotating body with angular velocity $\dot{\varphi}$. Therefore, if the angle that is transverse angular velocity $\omega(\mathrm{t})$ relative to rotating body coordinate system $\mathrm{x}$-axis is $\alpha(\mathrm{t})$, angle $\varphi(\mathrm{t})+\alpha(\mathrm{t})$ is relative to coordinate system $\mathrm{ox}_{0} \mathrm{y}_{0} \mathrm{z}$ (see Fig.7)'s $\mathrm{x}_{0}$-axis. Therefore, in order to obtain the transverse angular velocity relative to $\mathrm{ox}_{0} \mathrm{y}_{0} \mathrm{z}$, the value $\omega(\mathrm{t})$, azimuth $\alpha(\mathrm{t})$ and $\varphi(\mathrm{t})$ need to be obtained.

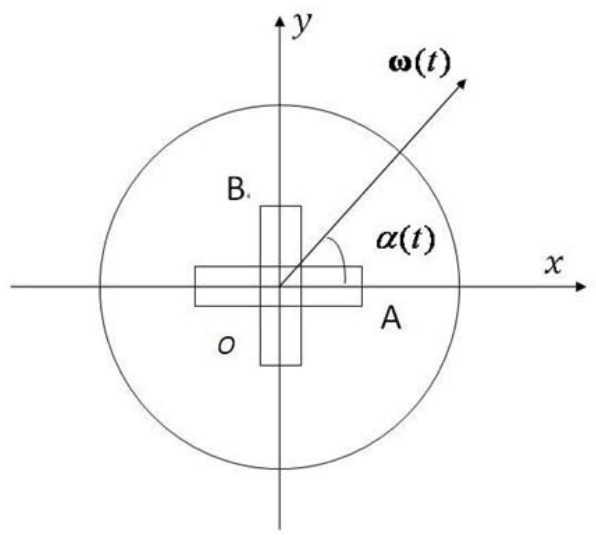


Fig.8 Schematic diagram of the installation location of the micro-mechanical pendulum

Method to obtain the transverse angular velocity of the rotating body coordinate system.As shown in Fig. 8, when any transverse angular velocity $\boldsymbol{\omega}(\mathbf{t})$ exists, with the reference coordinate system oxyz, the micro-mechanical pendulum A and B output voltage signal as follows:

$$
\begin{aligned}
& V_{A}(t)=k_{A} \omega(t) \sin \alpha(t) \\
& V_{B}(t)=k_{B} \omega(t) \cos \alpha(t)
\end{aligned}
$$

Eq. (4) and Eq. (5) are respectively output voltage signal for the micromechanical pendulum A and $\mathrm{B}$. The both of $\mathrm{k}_{\mathrm{A}}$ and $\mathrm{k}_{\mathrm{B}}$ are the scale factor of the $\mathrm{A}$ and $\mathrm{B}$. The $\alpha(\mathrm{t})$ is angle of transverse angular velocity $\omega(t)$ and $x$-axis. It is easy to find that the $V_{A}(t)$ and $V_{B}(t)$ are orthogonal (see Fig. 9). Using of orthogonal envelope detection method described in Fig. 10, we can obtain the size of transverse angular velocity and azimuth relative to coordinate system oxyz.

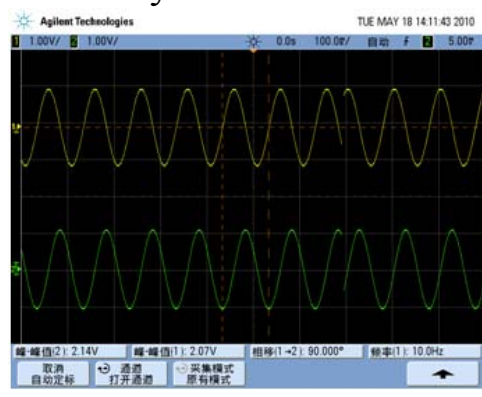

Fig.9

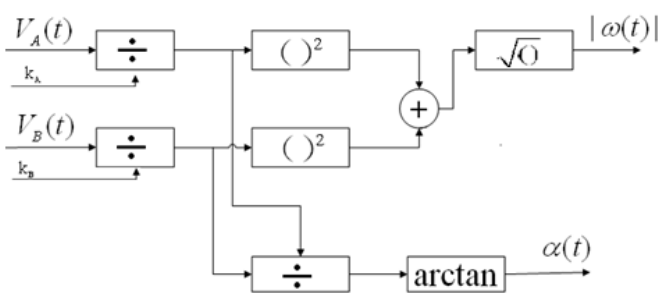

Fig.10 The principle of orthogonal envelope detection

With Eq. (4) and Eq. (5), solution is

$$
\begin{aligned}
& \boldsymbol{\omega}^{2}(t)=\left(V_{A}(t) / k_{A}\right)^{2}+\left(V_{B}(t) / k_{B}\right)^{2} \\
& \tan \alpha(t)=\left(V_{A}(t) k_{B}\right) /\left(V_{B}(t) k_{A}\right)
\end{aligned}
$$

With Eq. (6), transverse angular velocity is

$$
|\boldsymbol{\omega}(t)|=\sqrt{\left(V_{A}(t) / k_{A}\right)^{2}+\left(V_{B}(t) / k_{B}\right)^{2}}
$$

With Eq. (6), azimuth angle is

$$
\alpha(t)=\arctan \left(V_{A}(t) k_{B}\right) /\left(V_{B}(t) k_{A}\right)
$$

Therefore, Eq. (8) can determine the magnitude of the transverse angular velocity $\omega(\mathrm{t})$. Eq. (9) can determine the azimuth angle $\alpha(\mathrm{t})$.

If micro-mechanical pendulum $\mathrm{A}$ and $\mathrm{B}$ have the same scale factor that

$$
k_{\mathrm{A}}=k_{\mathrm{B}}=k
$$

Eq. (10) into Eq. (8) and (9), $\omega(\mathrm{t})$ is

$$
|\boldsymbol{\omega}(t)|=\sqrt{V_{A}^{2}(t)+V_{B}^{2}(t)} / k
$$


Azimuth's angle is

$$
\alpha(t)=\arctan V_{A}(t) / V_{B}(t)
$$

Therefore, for the same scale factor, the magnitude of the transverse angular velocity $\omega(t)$ is determined by Eq. (11) and the azimuth angle $\alpha(\mathrm{t})$ by Eq. (12).

The method to obtain spin angular velocity relative to the quasi-rotating body coordinate system.We adjust micro-mechanical pendulum $A$ and $B$ scale factor to the same. The angle $\boldsymbol{\varphi}(\mathbf{t})+\boldsymbol{\alpha}(\mathbf{t})$ between $\boldsymbol{\omega}(\mathbf{t})$ and $\mathbf{x}_{\mathbf{0}}$-axis is relative to quasi-rotating body coordinate system. It is supposed that the spin frequency of rotating body is $\mathbf{f}$, the azimuth angle caused by spin is always $\boldsymbol{\varphi}(\mathbf{t})=\mathbf{2} \boldsymbol{\pi f t}$ at $\mathrm{t}$-time. Because of $\dot{\boldsymbol{\varphi}}(\mathbf{t})=\mathbf{2} \boldsymbol{\pi f} \gg \dot{\boldsymbol{\alpha}}(\mathbf{t}), \boldsymbol{\varphi}(\mathbf{t})+\boldsymbol{\alpha}(\mathbf{t})$ can be represented by $\boldsymbol{\varphi}(\mathbf{t})=$ $\mathbf{2 \pi f}$. So Eq. (4) and (5) can be expressed by

$$
\begin{aligned}
& V_{A}(t)=k \omega(t) \sin (2 \pi f t) \\
& V_{B}(t)=k \omega(t) \cos (2 \pi f t)
\end{aligned}
$$

As can be seen from the above equation, voltage output signals $V_{A}(t)$ and $V_{B}(t)$ are proportional to the transverse angular velocity and scale factor is $\mathrm{k}$. The frequency modulated signal includes two main frequency of $f_{1}$ and $f_{2}$.

$$
\begin{aligned}
& f_{1}=f-f_{\omega} \\
& f_{2}=f+f_{\omega}
\end{aligned}
$$

In order to calculate the spin axis frequency at real-time, we need to eliminate frequency $f_{\omega}$ of low-frequency signal $\omega(\mathrm{t})$. In order to extract the carrier frequency we usually use orthogonal frequency detection method. The block diagram is shown in Fig. 11.

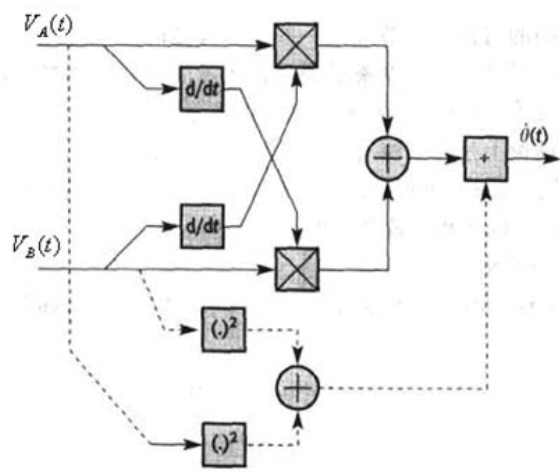

Fig.11 The principle of orthogonal frequency detection

Derivation of $V_{A}(t)$ and $V_{B}(t)$

$$
\begin{aligned}
& \dot{V}_{A}(t)=k \dot{\omega}(t) \sin (2 \pi f t)+k 2 \pi f \omega(t) \cos (2 \pi f t) \\
& \dot{V}_{B}(t)=k \dot{\omega}(t) \cos (2 \pi f t)-k 2 \pi f \omega(t) \sin (2 \pi f t)
\end{aligned}
$$




$$
\begin{aligned}
\dot{V}_{A}(t) V_{B}(t) & =k^{2} \boldsymbol{\omega}(t) \dot{\omega}(t) \sin (2 \pi f t) \cos (2 \pi f t) \\
& +k^{2} 2 \pi f \omega^{2}(t) \sin ^{2}(2 \pi f t) \\
V_{A}(t) \dot{V}_{B}(t) & =k^{2} \boldsymbol{\omega}(t) \dot{\boldsymbol{\omega}}(t) \sin (2 \pi f t) \cos (2 \pi f t) \\
& -k^{2} 2 \pi f \omega^{2}(t) \cos ^{2}(2 \pi f t)
\end{aligned}
$$

With Eq. (19) and (20), to solve

$$
\dot{V}_{A}(t) V_{B}(t)-V_{A}(t) \dot{V}_{B}(t)=k^{2} 2 \pi f \omega^{2}(t)
$$

Because of

$$
V_{A}^{2}(t)+V_{B}^{2}(t)=k^{2} \omega^{2}(t)
$$

When $V_{A}^{2}(t)+V_{B}^{2}(t) \neq 0$, Eq. (21)/Eq. (22) is

$$
\frac{\dot{V}_{A}(t) V_{B}(t)-V_{A}(t) \dot{V}_{B}(t)}{V_{A}^{2}(t)+V_{B}^{2}(t)}=2 \pi f
$$

Resulting in the spin frequency

$$
f=\frac{\dot{V}_{A}(t) V_{B}(t)-V_{A}(t) \dot{V}_{B}(t)}{2 \pi\left[V_{A}^{2}(t)+V_{B}^{2}(t)\right]}
$$

According to Eq. (24), spin frequency given shows eliminating the effects of transverse angular velocity frequency and resulting in the spin angular velocity $\dot{\varphi}=2 \pi \mathrm{f}$. As the above computation, all values are obtained in the same moment. So the delay is inevitable in the actual process of computation.

The method to obtain the yaw and pitch ragular velocity relative to the quasi-rotating body coordinate system. Using of the aforementioned methods for transverse angular velocity and spin angular velocity, we real-time obtain the value of the transverse angular velocity $\boldsymbol{\omega}(\mathbf{t})$ and azimuth angle $\boldsymbol{\alpha}(\mathbf{t})$ relative to the rotating body coordinate system, and spin angular velocity $\dot{\boldsymbol{\varphi}}$ relative to the quasi-rotating body coordinate system. According to the coordinate transformation (3), the yaw angular velocity $\boldsymbol{\omega}_{\mathbf{p h}}$ and pitch angular velocity $\boldsymbol{\omega}_{\mathbf{f y}}$ can be calculated for the quasi-rotating body's coordinate system $\mathbf{o x}_{\mathbf{0}} \mathbf{y}_{\mathbf{0}} \mathbf{z}$. As shown

$$
\begin{aligned}
& \boldsymbol{\omega}_{p h}=|\boldsymbol{\omega}(t)| * \cos (\alpha(t)+\varphi(t)) \\
& \boldsymbol{\omega}_{f y}=|\boldsymbol{\omega}(t)| * \sin (\alpha(t)+\varphi(t))
\end{aligned}
$$

$\alpha(\mathrm{t})$ is azimuth angle of the transverse angular velocity $\omega(\mathrm{t})$ relative to the rotating body coordinate system. $\varphi(t)$ is the angle turned by the rotating body

$$
\varphi(t)=\int_{0}^{t} \dot{\varphi}(t) d t
$$

On the time integral for Eq. (25) and Eq. (26), Available as yaw angle $\Omega_{\mathrm{ph}}$ and pitch angle $\Omega_{\mathrm{fy}}$ 


$$
\begin{gathered}
\Omega_{p h}=\int_{0}^{t} \boldsymbol{\omega}_{p h} d t \\
\Omega_{f y}=\int_{0}^{t} \boldsymbol{\omega}_{f y} d t
\end{gathered}
$$

\section{Conclusion}

Micromechanical pendulum mounted on a rotating body. Using of rotating body spin instead of the traditional gyro driving force generated by its own driver structure, the gyro driven by rotating carrier has been constituted. Carrier-driven gyro can sensitive rotating body's yaw, pitch and spin angular velocity at the same time. It has the function of the three traditional gyro.

Micro-mechanical pendulum and the rotating body together constitute the closed-loop gyro driven by rotating carrier. It is used to rotating body attitude control. This technology has not only broken through the bottleneck of rotating body attitude control, and also greatly reduced the cost of using.

\section{Acknowledgment}

The project has been funded by National Natural Science Foundation-funded project (60971024), The Beijing Natural Science Fund Project (4112020), Beijing Key Laboratory of Sensor Open Project (5026035209), and Ministry of Education Key Laboratory project of modern measurement and control technology. Thanks a lot.

\section{References}

[1]Fuxue Zhang, Wei Zhang, Silicon Micromechantcal Gyroscope, US 7,805,994 B2.

[2]Zhang Fuxue, Zhang Wei, Silicon micromechanlcal gyroscope without driving structrue, GB 2449955A.

[3]Fuxue Zhang, Hongwei Wang, Wei Zhang, Yu Liu, Carrier-driven silicon micromachined gyroscope, ZL 200410028228.X.

[4]Fuxue Zhang, Nan Zhang, Xu Mao, Silicon Micromachined Gyroscope for Resistance to Impact, ZL 200510134857.3.

[5]Fuxue Zhang, Nan Zhang, Wei Zhang, Xu Mao, Yu Liu, Two-dimensional silicon micromachined gyroscope, ZL 200510134858.8.

[6]Fuxue Zhang, Hongwei Wang, Wei Zhang, Xu Mao, Silicon Micromachined Gyroscope, ZL 200410029089.0.

[7]Fuxue Zhang, Wei Zhang, A New Silicon Micromachined Gyroscope, ZL 200710105849.5.

[8]Qingwen Yan, Fuxue Zhang, Wei Zhang, The Solution Software for Silicon Micro-machined Gyroscope Attitude, Computer software copyright registration certificate No.0157869. 